\title{
Melatonin-Loaded Nanoparticles for Enhanced Antidepressant Effects and HPA Hormone Modulation
}

\author{
Min Si, ${ }^{1}$ Qianshu Sun, ${ }^{1}$ Hongcheng Ding, ${ }^{1}$ Chengcheng Cao, ${ }^{2}$ Mingwei Huang, ${ }^{1}$ Qian Wang, \\ Hua Yang, ${ }^{3}$ and Yi Yao $\mathbb{1}^{1}$ \\ ${ }^{1}$ Department of Endocrine, Renmin Hospital, Hubei University of Medicine, Shiyan, Hubei 442000, China \\ ${ }^{2}$ Department of Internal Medicine, Guoyao Dongfeng Maojian Hospital, Shiyan, Hubei 442008, China \\ ${ }^{3}$ Department of Urology, Renmin Hospital, Hubei University of Medicine, Shiyan, Hubei 442000, China
}

Correspondence should be addressed to Yi Yao; 278764942@qq.com

Min Si and Qianshu Sun contributed equally to this work.

Received 8 January 2020; Revised 8 March 2020; Accepted 7 April 2020; Published 9 May 2020

Guest Editor: Mingqiang Li

Copyright ( 2020 Min Si et al. This is an open access article distributed under the Creative Commons Attribution License, which permits unrestricted use, distribution, and reproduction in any medium, provided the original work is properly cited.

\begin{abstract}
Background. The present work aims at formulating the melatonin-loaded nanoparticles (MTNPs) exhibiting the controlled-release and $\mathrm{pH}$-sensitivity to repurpose the use of melatonin in the treatment of depressive-like behaviors and hypothalamus-pituitaryadrenal (HPA) axis dysregulation. Methods. MTNPs were characterized for the size, drug incorporation, and in vitro release in the different $\mathrm{pH}$ environments. Its merits were in vivo tested on the pinealectomized rats presenting the depressive-like behaviors and the abnormal HPA axis activity by calculating the improvement on saccharin preference, swimming immobility time, and the negative feedback of HPA axis. Results. Results revealed that MTNPs showed nanometer size, $15.77 \%$ of drug loading, 33.82\% of encapsulation efficiency, the different controlled-release profiles in different $\mathrm{pH}$ environments ( $\mathrm{pH} 1.2$, $\mathrm{pH}$ 6.8, and $\mathrm{pH}$ 7.4), more sensitivity release in simulated intestinal fluid ( $\mathrm{pH}$ 7.4) and blood ( $\mathrm{pH}$ 6.8), and less sensitivity release in simulated gastric fluid ( $\mathrm{pH}$ 1.2). Furthermore, MTNPs displayed better antidepressant actions in reducing the immobility time of forced swimming test, increasing the preference for saccharin, and sensitizing the blunt negative feedback of HPA axis, when compared to the free melatonin. Conclusions. The controlled-release nanoparticles is shown to be an effective improvement on the dosage form for melatonin, which is worthy of futuristic and complete evaluation.
\end{abstract}

\section{Introduction}

The released report by the World Health Organization (WHO) in 2017 shows that depression has become the most widespread and burdensome mental illness [1]. There are currently about 350 million patients worldwide, and it is projected that depression will be the most popular disease in the developing countries by $2022[2,3]$. Melatonin (MT) is an endogenous bioactive substance which is mainly secreted by pineal gland [4]. Melatonin has a broad range of physiological activities, including body temperature regulation [5], nervous regulation [6], endocrine regulation [7], immune regulation [8], and hormone secretion [9]. Melatonin secretion decreases during the course of depression and increases after achieving remission, suggesting that the abnormality of melatonin plays a role in the pathogenesis of depression $[10,11]$. As the deep understanding of neuroendocrine therapy of depression, melatonin has been found to have a certain effect on improving depressive-like behaviors $[12,13]$. Boer et al. [14] reported that melatonin may be used as a novel antidepressant in clinical treatment of a variety of depression, especially in the treatment of major depressive disorder (MDD). Clinical studies showed that the plasma level of melatonin decreases in MDD patients [15]. In the preclinical studies evaluating the antidepression efficacy of melatonin, it was found that the immobility period of mice in the forced swimming test (FST) decreased in the dose-dependent manner after the daily administration of melatonin $(2.5-10 \mathrm{mg} / \mathrm{kg})$ in 3 to 6 
days [16]. The administration of melatonin by intraperitoneal $(0.1-30 \mathrm{mg} / \mathrm{kg})$ or intracerebroventricular (0.001-0.1 nmol/ site) route also reduced the immobility period in the tail suspension test (TST) [17]. Unpredictable chronic exposure to stress-induced high levels of serum corticosterone in mice; however, oral administration of exogenous melatonin for 5 weeks ( 1 and $10 \mathrm{mg} / \mathrm{kg}$ ) decreased the higher serum corticosterone levels [18]. These studies provided the direct evidences supporting the hypothesis that the melatonin acts as an antidepressant, and melatonin might have regulatory roles in the activity of hypothalamus-pituitary-adrenal (HPA) axis [19-22].

After the oral administration, the blood concentration of the melatonin increases rapidly and then decreases rapidly with a short half-life of about 60 minutes in the blood and a low bioavailability of about $15 \%$ [23]. The pharmaceutical dosage forms are useful in the precision and rationalization of drug delivery, so as to realize the sustained and stable drug release and the long-term effective blood drug concentration, to avoid the fluctuation of peak-valley concentration, and to reduce the frequency of administration [24]. In addition, the effective dosage forms can also reduce the side effects of drugs, such as the protection on gastrointestinal tract $[25,26]$. By using the polymer nanometer materials and the drug-loaded nanoparticle technology, the melatonin compound is wrapped and attached to the nanoparticle, so as to slow down the dissolution rate and improve the drug absorption in vivo [27-29]. The design of the dosage form for melatonin has been a new strategy to improve its curative effect in various diseases [30, 31].

In this study, the gelatin, polylactic acid (PLA), and chitosan were used to prepare melatonin-loaded nanoparticles (MTNPs). Then the effects on the depressive behaviors and the hormone secretion in vivo in the pinealectomized rats were compared between the administration of MTNPs and free melatonin.

\section{Material and Methods}

2.1. Preparation of Nanoparticles Loaded with Melatonin. The absolute ethyl alcohol solution containing melatonin $(0.5 \mathrm{~mL})$ and the aqueous solution containing gelatin $(0.5 \mathrm{~mL})$ were mixed to give a clear solution under $50^{\circ} \mathrm{C}$. Then the solution was dropped slowly into the dichloromethane solution containing PLA $(9.0 \mathrm{~mL})$ followed with $1 \mathrm{~h}$ stirring and $1 \mathrm{~h}$ ultrasonic vibration to obtain the white emulsion I. The final concentrations of melatonin, gelatin, and PLA were $0.4 \%, 0.1 \%$, and $1.2 \%$, respectively. Emulsion I was slowly dropped into the dichloromethane solution $(30 \mathrm{~mL})$ containing Span-80 and Tween-80, followed with $1 \mathrm{~h}$ stirring, subsequent heating at $39^{\circ} \mathrm{C}$ to evaporate $30 \mathrm{~mL}$ of dichloromethane, and $1 \mathrm{~h}$ ultrasonic vibration to obtain the yellow emulsion II $(10 \mathrm{~mL})$. Emulsion II was slowly dropped into the aqueous solution $(50 \mathrm{~mL})$ containing $0.25 \%$ chitosan and $1 \%$ acetic acid, followed with $1 \mathrm{~h}$ stirring and $1 \mathrm{~h}$ ultrasonic vibration to obtain the white emulsion III. Emulsion III was stirred to volatilize the organic solvent for $24 \mathrm{~h}$ at $30^{\circ} \mathrm{C}$ and then was centrifuged at $5000 \mathrm{rpm} / \mathrm{min}$ to obtain the precipitate. The precipitate was washed by deionized water, centrifuged for three times, and dried under vacuum decompression to obtain the product of melatonin-loaded nanoparticles (MTNPs). The procedure is schematically shown in Figure 1.

2.2. Measurement of Particle Size, PDI, and Morphology. The hydrodynamic diameter and polydispersity index (PDI) value of MTNPs were analyzed by dynamic light scattering (DLS). Five measurements were taken using the Zetasizer Nano analyzer from Malvern Instruments (Malvern, UK). Transmission electron microscopy (Philips CM12, Eindhoven, Netherlands) was applied to evaluate the morphology of MTNPs. The particle sizes of MTNPs were determined by measuring the diameters with the ImageJ measurement tool from a number of TEM images.

2.3. Determination of Drug Incorporation. One milligram per milliliter of MTNPs in dichloromethane was diluted for 100 times by $30 \%$ ethanol. Then the water phase was collected by stirring thoroughly and standing for $20 \mathrm{~min}$. The absorbance of the water phase was measured by a UV spectrophotometer at $278 \mathrm{~nm}$. The melatonin concentration was calculated according to the standard curve of melatonin at $278 \mathrm{~nm}$. The encapsulation efficiency (EE) and drug loading (DL) of melatonin were calculated by the equations (1) and (2), respectively, as

$$
\begin{aligned}
\text { Encapsulation efficiency }(\%) & =\frac{\text { Mass of melatonin in nanoparticles } \times 100 \%}{\text { Mass of initial melatonin used }} \\
\text { Drug loading }(\%) & =\frac{\text { Mass of melatonin in nanoparticles } \times 100 \%}{\text { Mass of nanoparticles }}
\end{aligned}
$$

2.4. In Vitro Release Study. The phosphate-buffered salines (PBS) of $\mathrm{pH} 7.4, \mathrm{pH} 6.8$, and $\mathrm{pH} 1.2$ were prepared and used for simulating blood, intestinal fluid, and gastric fluid, respectively. $10 \mathrm{~mL}$ of $0.1 \mathrm{mg} / \mathrm{mL}$ MTNPs was placed into a dialysis bag. The dialysis bag was put into $50 \mathrm{~mL}$ PBS with different $\mathrm{pH}$ and then was stirred magnetically at $37^{\circ} \mathrm{C}$. At the indicated interval, $1 \mathrm{~mL}$ of sample PBS was removed for analysis, and the new PBS ( $1 \mathrm{~mL}$ ) was supplemented. The absorbance of the sample PBS was measured by a UV spectrophotometer at $278 \mathrm{~nm}$ to plot the cumulative release curve. All the drug release studies were performed in triplicates.

2.5. Animals and Groups. Male Wistar rats were housed in a temperature-controlled room $\left(25^{\circ} \mathrm{C}\right)$ under the light/dark 


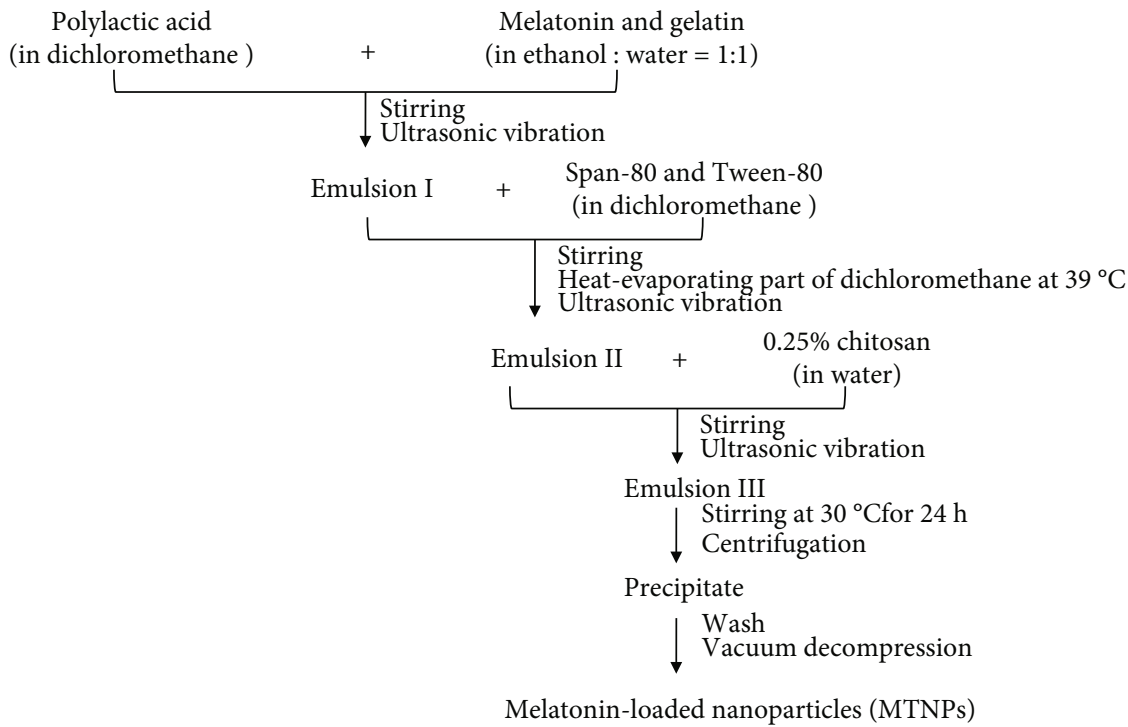

FIGURE 1: Schematic presentation of the preparation of melatonin-loaded nanoparticles (MTNPs).

cycle (12:12 h, lights on at 08:00 a.m.). Experimental procedures were approved by the Animal Ethics Committee of Hubei University of Medicine. The rats were randomly divided into four groups with 10 rats per group: the sham group, normal rats treated with vehicle; the Pin group, pinealectomized rats; the Pin-MT group, pinealectomized rats treated with free melatonin; and the Pin-MTNPs group, pinealectomized rats treated with MTNPs.

2.6. Surgery and Treatment. The surgery was performed following the method previously reported by Hoffmann and Reiter [32]. Briefly, the rats were anesthetized and adapted to a stereotactic apparatus, and then the hair on the top of the head was scraped and removed. Transverse incision was cut between two ears to expose the sagittal suture and lambdoidal suture. A piece of rectangular bone was peeled off from the dura using a pointed dental burr with the sagittal suture as the symmetry axis. After the dura was cut off, the sagittal venous sinus was ligated on two sides and was cut off at the middle, and then the pineal gland under the sagittal venous sinus was removed. After using the Gelfoam sponge to stem the bleeding, the rectangular bone was returned to its original position, and then the skin was sutured. The sham rats encountered the same procedure except that the pineal gland was removed. After 7 days of surgery, the rats could be used in the study.

Free melatonin or MTNPs, dissolved in saline containing $4 \%$ ethanol, was injected subcutaneously at 17:00 p.m. at the dose of $10 \mathrm{mg} \mathrm{kg}^{-1}$ and $60 \mathrm{mg} \mathrm{kg}^{-1}$, respectively. The doses ensured the equal amount of melatonin given to rats. The sham group and Pin group were injected the equal volume of saline subcutaneously. The administration was kept for 4 weeks.

2.7. Saccharin Preference Test. During the last four days of the administration, all rats were given two weighed bottles, one containing pure water and the other one containing $0.1 \%$ saccharin solution. After $24 \mathrm{~h}$, the two bottles were weighed to calculate the consumption of drinking water using the difference before and after detection. The consumption for 4 consecutive days was recorded, and then the taste preference was calculated by the equation (3) as

$$
\text { Preference for saccharin }(\%)=\frac{\text { Mass of saccharin solution } \times 100 \%}{\text { Total mass of drinking water }(\text { saccharin }+ \text { water })}
$$

2.8. Forced Swimming Test (FST). After $1 \mathrm{~h}$ of final administration, the rats in each group were put into a glass cylinder ( $\phi$ of $20 \mathrm{~cm}$, height of $40 \mathrm{~cm}$ ) filled with $24 \mathrm{~cm}$ of water at $25^{\circ} \mathrm{C}$. Each rat was allowed to freely swim for $6 \mathrm{~min}$, and the immobility time was recorded in the last $4 \mathrm{~min}$. The water was replaced before each test to avoid the interference of fecal and odor from each rat.
2.9. Hormone Assay. Before the dexamethasone (Dex) challenge, the blood of each rat was collected and the basal level of corticosterone (CORT) was detected by ELISA. Then Dex $(30 \mathrm{mg} / \mathrm{kg})$ was injected intraperitoneally to inhibit the secretion of CORT in vivo. After $2 \mathrm{~h}$ of injection, the blood sample was collected and the CORT level was measured again. 
2.10. Statistical Analyses. All statistical analyses were performed using SPSS (version 12.0.1, SPSS Inc., Chicago, IL, United States). Data are expressed as the means \pm SD, and $P<0.05$ was considered statistically significant. One-way ANOVA was used followed by the post hoc test for group difference.

\section{Result}

3.1. Basic Characteristics of the MTNPs. The hydrodynamic particle size of MTNPs was $96.12 \pm 13.53 \mathrm{~nm}$, and the PDI value was $0.203 \pm 0.01$. The TEM image showed the morphology of MTNPs as the spherical shape (Figure 2). Histograms indicated the particle size distribution of MTNPs (Figure 3). MTNPs had the particle size distribution between 23 and $106 \mathrm{~nm}$, and the average size was $54.71 \pm 17.29 \mathrm{~nm}$. The particle size between 39.45 and $55.90 \mathrm{~nm}$ accounted for $51.6 \%$ and was the relatively concentrated size range.

3.2. Encapsulation Efficiency and Drug Loading of MTNPs. The drug loading of MTNPs produced from 3 batches was $15.77 \pm 1.74 \%$ in Figure 4 . The encapsulation efficiency from 3 batches was $33.82 \pm 0.53 \%$.

3.3. In Vitro Release Profiles of MTNPs in Different $p H$ Solutions. The release kinetics of melatonin was evaluated in three different in vitro environments $(\mathrm{pH} 1.2$, simulated gastric fluid; $\mathrm{pH} 6.8$, simulated intestinal fluid; and $\mathrm{pH} 7.4$, simulated blood). In the simulated gastric fluid ( $\mathrm{pH} 1.2)$, the melatonin release rate was the slowest and less than $20.3 \%$ at $8 \mathrm{~h}$ (Figure 5(a)). In the simulated intestinal environment ( $\mathrm{pH}$ 6.8) and blood $(\mathrm{pH} 7.4)$, the degradation of PLA and chitosan was accelerated, so as that melatonin release rates increased to $81.4 \%$ (Figure 5(b)) and $96.7 \%$ (Figure 5(c)) at $8 \mathrm{~h}$, respectively.

3.4. Effect of MTNPs on Saccharin Preference in the Pinealectomized Rats. The preference for saccharin is an indicator for evaluating anhedonia. As shown in Figure 6, the preference for saccharin of each group was similar before pinealectomy $(P>0.05)$. After pinealectomy, the rats of the Pin group showed statistically significantly decreased preference for saccharin $(P<0.05)$, indicating that the pinealectomized rats have the depressive-like behavior. After the various administrations, both MT and MTNPs could increase the preference for saccharin in the pinealectomized rats. As compared to the MT group, the preference for the saccharin of rats in the MTNPs group was higher, but no significant statistical significance was observed.

3.5. Effect of MTNPs in FST. As shown in Figure 7, at week 0, all groups did not present the notably difference of the immobility time $(P>0.05)$. After the pinealectomy and administration for 4 weeks, the significant difference of the immobility time between the Pin group and sham group was shown $(P<0.001)$, representing the induced depression to rats. However, both treatment groups with MT and MTNPs significantly reduced the immobility time in FST compared to the Pin group. Moreover, the FST result showed

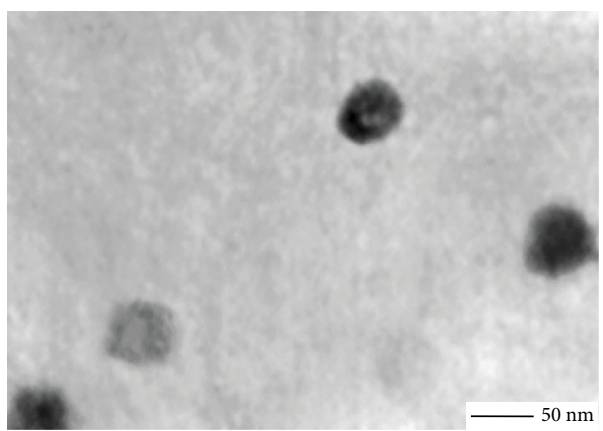

FIgURE 2: Transmission electron microscope image of melatoninloaded nanoparticles (MTNPs).

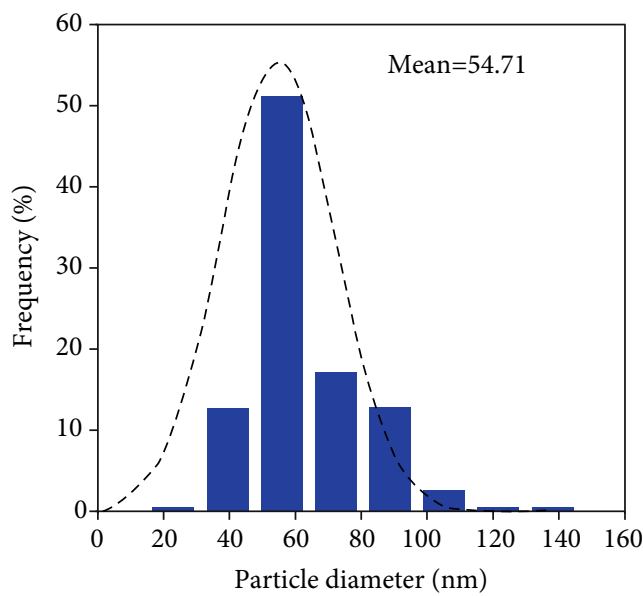

FIgURE 3: The particle size distribution for melatonin-loaded nanoparticles (MTNPs).

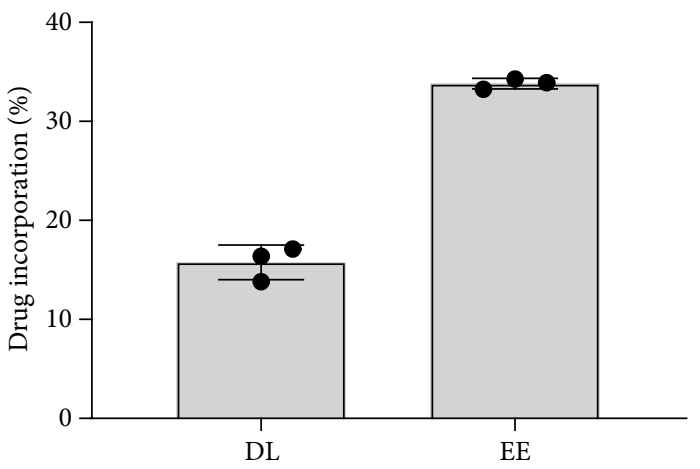

FIgURE 4: Drug loading and encapsulation efficiency of melatoninloaded nanoparticles (MTNPs). DL: drug loading; EE: encapsulation efficiency.

that MTNPs exhibited an improvement more significantly to $\operatorname{MT}(P<0.05)$.

3.6. Effect of MTNPs in HPA Axis Activity. Plasma CORT levels in basal condition (before the Dex injection) and after the Dex injection were observed (Figure 8). Before the Dex injection, the basal plasma levels of CORT of the pinealectomized rats were higher than that of sham rats, but there was no statistical difference $(P>0.05)$. After the Dex injection, 


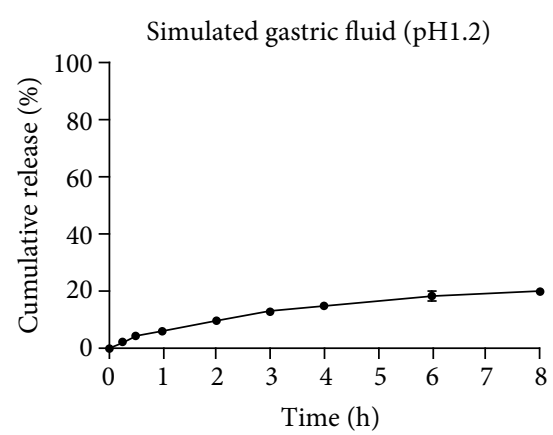

(a)

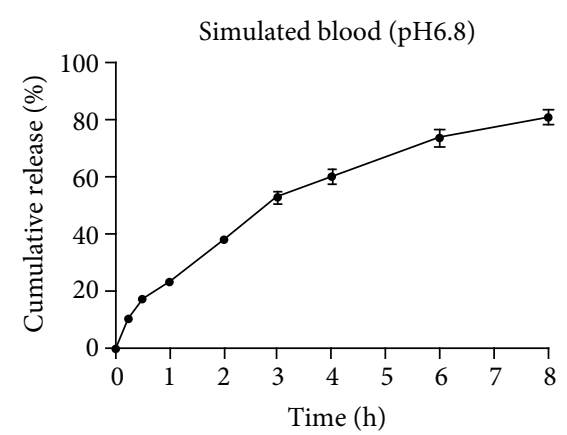

(b)

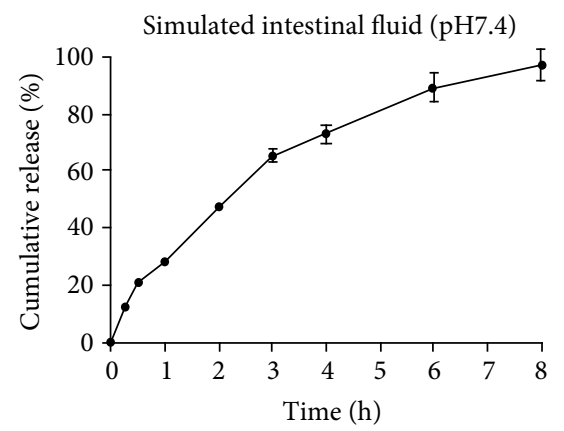

(c)

FIGURE 5: In vitro release profile of melatonin-loaded nanoparticles (MTNPs). (a) pH 1.2, simulated gastric fluid. (b) pH 6.8, simulated blood. (c) $\mathrm{pH} 7.4$, simulated intestinal fluid.

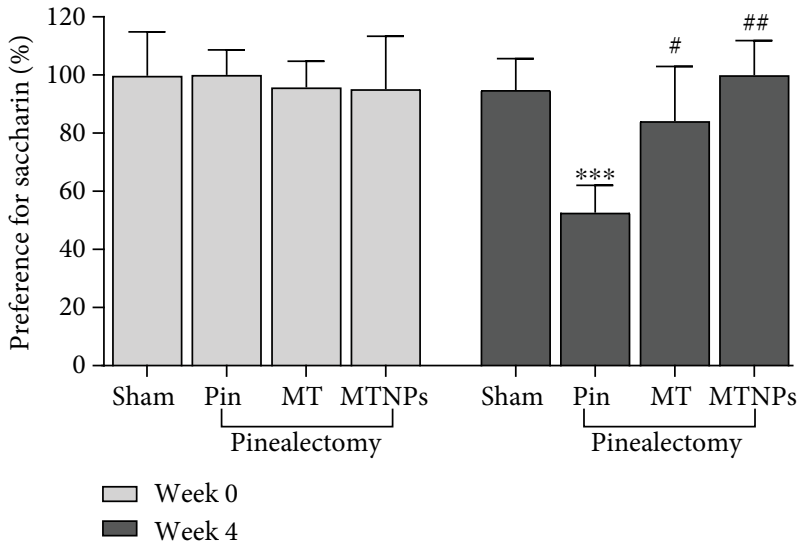

FIGURE 6: Effect of melatonin-loaded nanoparticles (MTNPs) on saccharin preference. ${ }^{* * *} P<0.001$ vs. the sham group; ${ }^{\#} P<0.05$ and ${ }^{\#} P<0.01$ vs. the Pin group. Pin: pinealectomized rats; MT: melatonin.

the plasma CORT levels of all rats decreased significantly $(P<0.05)$, presenting the negative feedback mechanism of the HPA axis. When analyzing the CORT levels, it was observed that the CORT level of the Pin group was significantly higher than that of the sham group $(P<0.05)$. When compared with the Pin group, the CORT level of the MT group showed a decreased tendency without statistical difference $(P>0.05)$; however, the CORT level of the MTNP group significantly decreased $(P<0.05)$. When analyzing the decreased fold of the CORT level, it was observed that the decreased fold of the Pin group was significantly lower

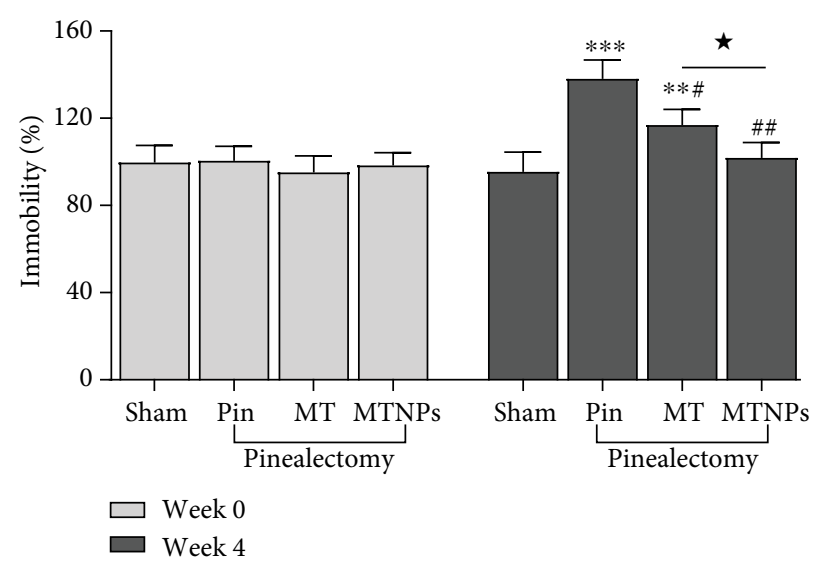

Figure 7: Effect of melatonin-loaded nanoparticles (MTNPs) on the immobility time in forced swimming test. ${ }^{* *} P<0.01$ and ${ }^{* * *} P<$ 0.001 vs. the sham group; ${ }^{\#} P<0.05$ and ${ }^{\# \#} P<0.01$ vs. the Pin group; ${ }^{\star} P<0.05$ vs. the MT group. Pin: pinealectomized rats; MT: melatonin.

than that of the sham group, indicating the pinealectomy impaired the negative feedback of the HPA axis. Both rats in the MT group and MTNP group showed a notable decrease in the CORT level and significant increased change fold $(P<0.05)$, indicating that MT and MTNPs could improve the impaired negative feedback. When compared with the decrease fold of CORT in the MT group, the decrease fold in the MTNP group was significantly higher $(P<0.05)$, suggesting that MTNPs improved the damaged HPA axis more than MT. 


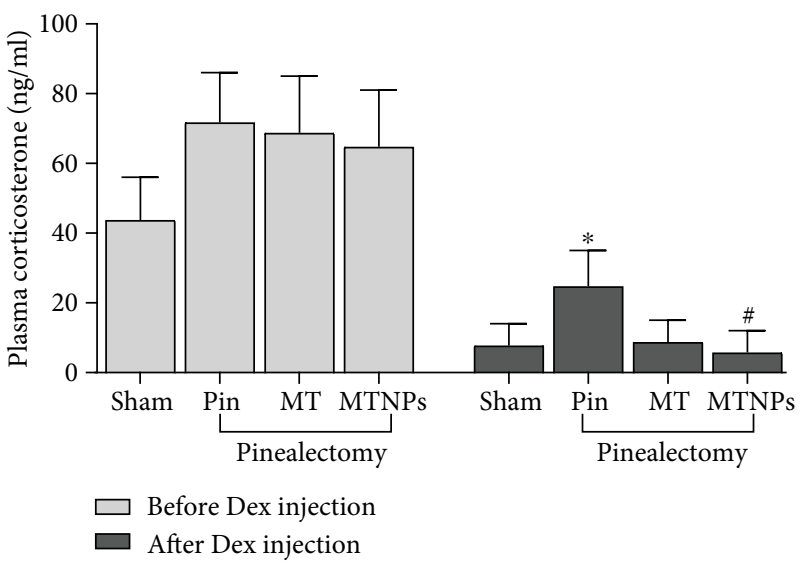

(a)

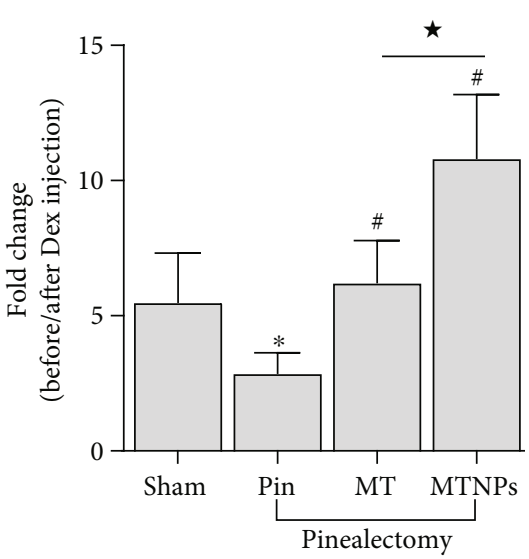

(b)

FIGURE 8: Effect of melatonin-loaded nanoparticles (MTNPs) on the plasma corticosterone level. (A) The plasma corticosterone level. (b) The decreased fold change of plasma corticosterone. ${ }^{*} P<0.05$ vs. the sham group; ${ }^{\#} P<0.05$ vs. the Pin group; ${ }^{\star} P<0.05$ vs. the MT group. Pin: pinealectomized rats; MT: melatonin.

\section{Discussion}

Although melatonin is metabolized rapidly in vivo and has a short half-life of about 60 minutes [23], the controlledrelease dosage forms can delay the elimination time of melatonin residue in vivo, prolong the retention time, and maintain the physiological effect of melatonin. There are various carriers used to prepare the controlled-release dosage forms of melatonin, such as silica [33], diamine polymer [33], hydroxypropyl methylcellulose phthalate [34], alginate [35], and poly(lactic-co-glycolic acid) (PLGA) [28, 36]. In the study, PLA and chitosan are used as the controlled-release carriers for melatonin, and gelatin is used as dispersant. The hydrophilic gelatin is nontoxic and nonantigenic protein and plays the role of emulsifying and stabilizing agent. PLA has good biodegradability, good biocompatibility [37, 38], and no toxicity [39]. PLA is hydrolyzed fully to lactic acid, the natural substance of human body, which in turn is converted into carbon dioxide and is expelled from the lung $[40,41]$. Chitosan is a natural poly(glucosamine) with good biocompatibility, biodegradability [42], and bioactivities [43]. According to the characteristics of the polymers, the technique of emulsification-solvent evaporation is adopted to prepare the melatonin-loaded nanoparticles (MTNPs). The melatonin compound is adsorbed, embedded, and attached by PLA and chitosan, rather than chemically linked to these polymers, thus not destroying the nature of melatonin. In the process of drug production, encapsulation efficiency and particle size are the two most essential features. The purpose of the drug determines the particle size. The particle size of MTNPs is $54.71 \mathrm{~nm}$, lower than $100 \mathrm{~nm}$, which is favorable for the feasible purpose of penetrating the blood-brain barrier. However, the encapsulation efficiency of MTNPs is low. The improvement of encapsulation efficiency might be adjusted from the stirring speed, the polymers concentration, and the solvent evaporation temperature. The process of the controlled-release [44] of melatonin from MTNPs firstly displays as the detachment and diffusion of melatonin adsorbed on the surface of MTNPs. Secondly, PLA and chitosan which are located at the outer layer of MTNPs proceed swelling, and the swelling causes a plurality of micropores on the surface of MTNPs. These actions promote the gradual dissolution and release of melatonin through the micropores and also promote the permeation of solvent into the inside of MTNPs through the micropores and thereby accelerate again the dissolution and release of melatonin. Finally, the carrier materials of MTNPs are degraded and eroded, so that the melatonin is completely released. This process plays the role in maintaining the controlled release of melatonin.

It has been reported in literatures that melatonin has binding sites in the gastrointestinal tract of mammals, and the density of these binding sites differs in different regions of the intestines. Lee et al. demonstrated that the binding site density follows the descending order: ileum, jejunum $>$ duodenum, colon $>$ cecum $>$ esophagus [45]. Poon et al. found that the binding sites of human body are mainly located in the colon, cecum, appendix, and few ileus [46]. Bubenik et al. [47] compared that the binding site density in the intestines is overall higher than that in the stomach. In the present study, according to the tissue distribution characteristics of melatonin binding sites, we prepared the MTNPs with the slow release of melatonin in the stomach and the rapid release in the intestines. The melatonin can be rapidly absorbed along with the mass release of melatonin in the intestinal tissue because of the large absorption surface area of the intestinal tissue. Thereby, this action could improve the bioavailability of melatonin. Due to the removal of the pineal gland, the in vivo level of melatonin is much lower. The controlled release of melatonin can maintain the retention time of melatonin in vivo and keep the drug effect for a long time; therefore it plays a part in simulating the physiological function of pineal gland. 
Delagrange et al. [48] showed that melatonin and its receptor agonists are antidepressants. The plasma level of melatonin in the first-episode depression patients was lower than that in healthy people during the onset of depression and was also significantly lower than that in first-episode schizophrenia patients. This suggested that the decline of melatonin level is particularly closely related to the depression. Delagrange et al. also demonstrated that the plasma level of melatonin increases significantly as the depressive symptoms remission after treatment with antidepressants. In clinical trials, it is also reported that the serum level of melatonin was abnormal in MDD patients, although the level does not distinguish the severity of depression [49]. Oral administration with exogenous melatonin is useful for treating depression. Melatonin has an antidepressant effect on mouse undergoing the repeated forced swimming tests [16]. Melatonin also has the function in improving the mood of MDD patients and has good safety, good tolerance, and no adverse side effects [50]. In our study, it demonstrated that the pinealectomy produces depressive-like behaviors in rats, including significantly reducing saccharin preference in Wistar rats and prolonging the swimming immobility time. These indexes could be effectively improved by administration of exogenous melatonin [51]. Our results are consistent with the reported results. Furthermore, compared to the effects of free melatonin, MTNPs are more effective on the shortening of the immobility time and show a tendency to increase the saccharin preference, suggesting a better drug effect by MTNPs. It is speculated that this better improvement might be due to the controlled release of melatonin from MTNPs, which in turn maintains the plasma level for a long time.

Previous studies have shown that the melatonin has the inhibitory effect on the hypothalamus-pituitary-target axis activity (gonad, thyroid, and adrenal) [52, 53]. Moreover, melatonin also has an improved effect on the immune system, which causes the changes of cytokine secretion and regulates neuroendocrine. This makes the effect of melatonin more diverse and complicated on neuroendocrine, especially in many inconsistent results on the HPA axis [52]. For example, the reduction of melatonin caused by the pinealectomy has no significant effect on the CORT secretion in male rats [54], while the secretion in female rats is upregulated [55]. In the present study, the deficit of melatonin due to the pinealectomy showed a tendency to enhance the basal plasma CORT levels before Dex injection although there was no statistical difference among the four groups. However, after the Dex injection, the decrease fold of the CORT level in the Pin group was significantly less than that in the sham group, indicating that the absence of the pineal gland destroys the plastic change of the HPA axis activity. The higher CORT level in the pinealectomized rats is also consistent with their symptoms of depressive-like behaviors. The administration of the exogenous-free melatonin or MTNPs could augment the decrease folds of CORT after the Dex injection, make the plasticity of HPA axis sensitive, and improve the negative feedback of the HPA axis. Moreover, MTNPs could improve the negative feedback mechanism more than that of the free melatonin, which proves that the long-term release of melatonin might help to alleviate the overall decrease of melatonin caused by pinealectomy.

\section{Conclusion}

In the current work, the controlled-release nanoparticles loaded with melatonin in the treatment of pinealectomized rats were proven. These nanoparticles can effectively encapsulate melatonin and protect melatonin from degradation. The melatonin-loaded nanoparticles present both $\mathrm{pH}$ sensitivity and the controlled release. It helps the rapid release and absorption in the intestinal tissue to improve melatonin bioavailability. Then the in vivo results prove that the nanoparticles treatment effectively improve the depression-like behaviors and the HPA axis hormone secretion in the pinealectomized rats. The study however is in its infancy, and it still requires the optimization of encapsulation efficiency and needs the complete drug efficacy evaluation using the chronic unpredictable mild stress-induced rats.

\section{Data Availability}

All raw data used and analyzed during the current study can be available from the corresponding author on reasonable request.

\section{Conflicts of Interest}

The authors have no conflicts of interest regarding the publication of this paper.

\section{Authors' Contributions}

Min Si and Qianshu Sun contributed equally to this work.

\section{References}

[1] GBD 2017 Disease and Injury Incidence and Prevalence Collaborators, "Global, regional, and national incidence, prevalence, and years lived with disability for 354 diseases and injuries for 195 countries and territories, 1990-2017: a systematic analysis for the Global Burden of Disease Study 2017," The Lancet, vol. 392, no. 10159, pp. 1789-1858, 2018.

[2] "Depression and Other Common Mental Disorders Global Health Estimates," 2017, https://www.who.int/mental_ health/management/depression/prevalence_global_health_ estimates/en/.

[3] N. Sartorius, "Depression and diabetes," Dialogues in Clinical Neuroscience, vol. 20, no. 1, pp. 47-52, 2018.

[4] K. Karolczak and C. Watala, "The mystery behind the pineal gland: melatonin affects the metabolism of cholesterol," Oxidative Medicine and Cellular Longevity, vol. 2019, Article ID 4531865, 8 pages, 2019.

[5] R. Lok, M. J. van Koningsveld, M. C. M. Gordijn, D. G. M. Beersma, and R. A. Hut, "Daytime melatonin and light independently affect human alertness and body temperature," Journal of Pineal Research, vol. 67, no. 1, article e12583, 2019.

[6] B. S. Alghamdi, "The neuroprotective role of melatonin in neurological disorders," Journal of Neuroscience Research, vol. 96, no. 7, pp. 1136-1149, 2018.

[7] I. Mishra, R. M. Knerr, A. A. Stewart, W. I. Payette, M. M. Richter, and N. T. Ashley, "Light at night disrupts diel patterns of cytokine gene expression and endocrine profiles in zebra 
finch (Taeniopygia guttata)," Scientific Reports, vol. 9, no. 1, p. $15833,2019$.

[8] N. Ma, J. Zhang, R. J. Reiter, and X. Ma, "Melatonin mediates mucosal immune cells, microbial metabolism, and rhythm crosstalk: a therapeutic target to reduce intestinal inflammation," Medicinal Research Reviews, vol. 40, no. 2, pp. 606$632,2020$.

[9] E. Scarinci, A. Tropea, G. Notaristefano et al., "'Hormone of darkness" and human reproductive process: direct regulatory role of melatonin in human corpus luteum," Journal of Endocrinological Investigation, vol. 42, no. 10, pp. 1191-1197, 2019.

[10] M. Fava, S. D. Targum, A. A. Nierenberg et al., "An exploratory study of combination buspirone and melatonin SR in major depressive disorder (MDD): a possible role for neurogenesis in drug discovery," Journal of Psychiatric Research, vol. 46, no. 12, pp. 1553-1563, 2012.

[11] M. Valdés-Tovar, R. Estrada-Reyes, H. Solís-Chagoyán et al., "Circadian modulation of neuroplasticity by melatonin: a target in the treatment of depression," British Journal of Pharmacology, vol. 175, no. 16, pp. 3200-3208, 2018.

[12] E. H. Taniguti, Y. S. Ferreira, I. J. V. Stupp et al., "Neuroprotective effect of melatonin against lipopolysaccharide-induced depressive-like behavior in mice," Physiology \& Behavior, vol. 188, pp. 270-275, 2018.

[13] J. Liu, S. J. Clough, and M. L. Dubocovich, "Role of the MT1and MT2melatonin receptors in mediating depressiveand anxiety-like behaviors in $\mathrm{C} 3 \mathrm{H} / \mathrm{HeN}$ mice," Genes, Brain, and Behavior, vol. 16, no. 5, pp. 546-553, 2017.

[14] J. A. den Boer, F. J. Bosker, and Y. Meesters, "Clinical efficacy of agomelatine in depression: the evidence," International Clinical Psychopharmacology, vol. 21, Supplement 1, pp. S21S24, 2006.

[15] J. M. Bumb, F. Enning, J. K. Mueller et al., "Differential melatonin alterations in cerebrospinal fluid and serum of patients with major depressive disorder and bipolar disorder," Comprehensive Psychiatry, vol. 68, pp. 34-39, 2016.

[16] V. Raghavendra, G. Kaur, and S. K. Kulkarni, “Anti-depressant action of melatonin in chronic forced swimming-induced behavioral despair in mice, role of peripheral benzodiazepine receptor modulation," European Neuropsychopharmacology, vol. 10, no. 6, pp. 473-481, 2000.

[17] M. Mantovani, R. Pertile, J. B. Calixto, A. R. Santos, and A. L. Rodrigues, "Melatonin exerts an antidepressant-like effect in the tail suspension test in mice: evidence for involvement of $\mathrm{N}$-methyl-D-aspartate receptors and the L-arginine-nitric oxide pathway," Neuroscience Letters, vol. 343, no. 1, pp. 1-4, 2003.

[18] B. C. Detanico, A. L. Piato, J. J. Freitas, F. L. Lhullier, M. P. Hidalgo, and W. Caumo, "Antidepressant-like effects of melatonin in the mouse chronic mild stress model," European Journal of Pharmacology, vol. 607, no. 1-3, pp. 121-125, 2009.

[19] M. Garrido, J. Espino, D. González-Gómez et al., “The consumption of a Jerte Valley cherry product in humans enhances mood, and increases 5-hydroxyindoleacetic acid but reduces cortisol levels in urine," Experimental Gerontology, vol. 47, no. 8, pp. 573-580, 2012.

[20] Y. H. Wu, J. N. Zhou, R. Balesar et al., "Distribution of MT1 melatonin receptor immunoreactivity in the human hypothalamus and pituitary gland: colocalization of MT1 with vasopressin, oxytocin, and corticotropin-releasing hormone," The Journal of Comparative Neurology, vol. 499, no. 6, pp. 897910, 2006.
[21] A. Cagnacci, R. Soldani, and S. S. Yen, "Melatonin enhances cortisol levels in aged but not young women," European Journal of Endocrinology, vol. 133, no. 6, pp. 691-695, 1995.

[22] A. Turi, F. Di Prospero, O. Marasca, C. Romanini, F. Foschi, and G. Campanari, "Pineal gland hypothalamus-pituitaryadrenal axis relationships," Acta Europaea Fertilitatis, vol. 17, no. 3, pp. 205-206, 1986.

[23] R. L. DeMuro, A. N. Nafziger, D. E. Blask, A. M. Menhinick, and J. S. Bertino Jr., "The absolute bioavailability of oral melatonin," Journal of Clinical Pharmacology, vol. 40, no. 7, pp. 781-784, 2000.

[24] H. L. Gao and X. G. Jiang, "The progress of novel drug delivery systems," Yao Xue Xue Bao, vol. 52, no. 2, pp. 181-188, 2017.

[25] C. Andrade, "Sustained-release, extended-release, and other time-release formulations in neuropsychiatry," The Journal of Clinical Psychiatry, vol. 76, no. 8, pp. e995-e999, 2015.

[26] S. Talkar, S. Dhoble, A. Majumdar, and V. Patravale, "Transmucosal nanoparticles: toxicological overview," Advances in Experimental Medicine and Biology, vol. 1048, pp. 37-57, 2018.

[27] F. Blažević, T. Milekić, M. D. Romić et al., "Nanoparticlemediated interplay of chitosan and melatonin for improved wound epithelialisation," Carbohydrate Polymers, vol. 146, pp. $445-454,2016$.

[28] D. Ç. Altındal and M. Gümüşderelioğlu, "Melatonin releasing PLGA micro/nanoparticles and their effect on osteosarcoma cells," Journal of Microencapsulation, vol. 33, no. 1, pp. 5363, 2016.

[29] A. Priprem, J. R. Johns, S. Limsitthichaikoon, W. Limphirat, P. Mahakunakorn, and N. P. Johns, "Intranasal melatonin nanoniosomes: pharmacokinetic, pharmacodynamics and toxicity studies," Therapeutic Delivery, vol. 8, no. 6, pp. 373-390, 2017.

[30] J. Rybka, K. Kędziora-Kornatowska, D. Kupczyk, M. Muszalik, M. Kornatowski, and J. Kędziora, "Antioxidant effect of immediate- versus sustained-release melatonin in type 2 diabetes mellitus and healthy controls," Drug Delivery, vol. 23, no. 3, pp. 814-817, 2016.

[31] S. Hatem, M. Nasr, N. H. Moftah, M. H. Ragai, A. S. Geneidi, and S. A. Elkheshen, "Clinical cosmeceutical repurposing of melatonin in androgenic alopecia using nanostructured lipid carriers prepared with antioxidant oils," Expert Opinion on Drug Delivery, vol. 15, no. 10, pp. 927-935, 2018.

[32] R. A. Hoffman and R. J. Reiter, "Rapid pinealectomy in hamsters and other small rodents," The Anatomical Record, vol. 153, no. 1, pp. 19-21, 1965.

[33] A. M. Khattabi, W. H. Talib, and D. A. Alqdeimat, "The effect of polymer length on the in vitro characteristics of a drug loaded and targeted silica nanoparticles," Saudi Pharmaceutical Journal, vol. 26, no. 7, pp. 1022-1026, 2018.

[34] Y. Li, X. Zhao, Y. Zu et al., "Melatonin-loaded silica coated with hydroxypropyl methylcellulose phthalate for enhanced oral bioavailability: preparation, and in vitro-in vivo evaluation," European Journal of Pharmaceutics and Biopharmaceutics, vol. 112, pp. 58-66, 2017.

[35] E. E. Uchendu and E. R. Joachim Keller, "Melatonin-loaded alginate beads improve cryopreservation of yam (Dioscorea alata and D. cayenensis)," Cryo Letters, vol. 37, no. 2, pp. 7787, 2016.

[36] L. G. Martins, N. M. Khalil, and R. M. Mainardes, "PLGA nanoparticles and Polysorbate-80-coated PLGA nanoparticles 
increase the in vitro antioxidant activity of melatonin," Current Drug Delivery, vol. 15, no. 4, pp. 554-563, 2018.

[37] S. Farah, D. G. Anderson, and R. Langer, "Physical and mechanical properties of PLA, and their functions in widespread applications-a comprehensive review," Advanced Drug Delivery Reviews, vol. 107, pp. 367-392, 2016.

[38] K. S. Anderson, K. M. Schreck, and M. A. Hillmyer, "Toughening polylactide," Polymer Reviews, vol. 48, no. 1, pp. 85-108, 2008.

[39] B. Tyler, D. Gullotti, A. Mangraviti, T. Utsuki, and H. Brem, "Polylactic acid (PLA) controlled delivery carriers for biomedical applications," Advanced Drug Delivery Reviews, vol. 107, pp. 163-175, 2016.

[40] F. Mohamed and C. F. van der Walle, "Engineering biodegradable polyester particles with specific drug targeting and drug release properties," Journal of Pharmaceutical Sciences, vol. 97, no. 1, pp. 71-87, 2008.

[41] J. Liu and Y. Liu, "Preparation and application of polylactide," Sheng Wu Yi Xue Gong Cheng Xue Za Zhi, vol. 18, no. 2, pp. 285-7, 295, 2001, 295.

[42] B. R. Rizeq, N. N. Younes, K. Rasool, and G. K. Nasrallah, "Synthesis, bioapplications, and toxicity evaluation of chitosan-based nanoparticles," International Journal of Molecular Sciences, vol. 20, no. 22, p. 5776, 2019.

[43] F. Liaqat and R. Eltem, "Chitooligosaccharides and their biological activities: a comprehensive review," Carbohydrate Polymers, vol. 184, pp. 243-259, 2018.

[44] M. A. Mohammed, J. T. M. Syeda, K. M. Wasan, and E. K. Wasan, "An overview of chitosan nanoparticles and its application in non-parenteral drug delivery," Pharmaceutics, vol. 9, no. 4, p. 53, 2017.

[45] P. P. Lee and S. F. Pang, "Melatonin and its receptors in the gastrointestinal tract," Biological Signals, vol. 2, no. 4, pp. 181-193, 1993.

[46] A. M. Poon, P. H. Chow, A. S. Mak, and S. F. Pang, "Autoradiographic localization of 2[125I]iodomelatonin binding sites in the gastrointestinal tract of mammals including humans and birds," Journal of Pineal Research, vol. 23, no. 1, pp. 5$14,1997$.

[47] G. A. Bubenik, S. F. Pang, R. R. Hacker, and P. S. Smith, "Melatonin concentrations in serum and tissues of porcine gastrointestinal tract and their relationship to the intake and passage of food," Journal of Pineal Research, vol. 21, no. 4, pp. 251-256, 1996.

[48] P. Delagrange, J. Atkinson, and J. A. Boutin, "Therapeutic perspectives for melatonin agonists and antagonists," Journal of Neuroendocrinology, vol. 15, no. 4, pp. 442-448, 2003.

[49] J. Rabe-Jabłońska and A. Szymańska, "Diurnal profile of melatonin secretion in the acute phase of major depression and in remission," Medical Science Monitor, vol. 7, no. 5, pp. 946-952, 2001.

[50] M. A. Serfaty, D. Osborne, M. J. Buszewicz, R. Blizard, and P. W. Raven, "A randomized double-blind placebocontrolled trial of treatment as usual plus exogenous slowrelease melatonin $(6 \mathrm{mg})$ or placebo for sleep disturbance and depressed mood," International Clinical Psychopharmacology, vol. 25, no. 3, pp. 132-142, 2010.

[51] S. Moyanova, A. De Fusco, I. Santolini et al., “Abnormal hippocampal melatoninergic system: a potential link between absence epilepsy and depression-like behavior in WAG/Rij rats?," International Journal of Molecular Sciences, vol. 19, no. 7, p. 1973, 2018.

[52] I. Kostoglou-Athanassiou, D. F. Treacher, M. J. Wheeler, and M. L. Forsling, "Melatonin administration and pituitary hormone secretion," Clinical Endocrinology, vol. 48, no. 1, pp. 31-37, 1998.

[53] R. Konakchieva, Y. Mitev, O. F. Almeida, and V. K. Patchev, "Chronic melatonin treatment and the hypothalamopituitary-adrenal axis in the rat: attenuation of the secretory response to stress and effects on hypothalamic neuropeptide content and release," Biology of the Cell, vol. 89, no. 9, pp. 587-596, 1997.

[54] M. E. Diatroptov, S. M. Slesarev, and E. V. Slesareva, "Characteristics of 4-day Infradian biorhythms in mature male Wistar rats after pinealectomy," Bulletin of Experimental Biology and Medicine, vol. 163, no. 1, pp. 109-113, 2017.

[55] T. F. Ogle and J. I. Kitay, "Effects of pinealectomy on adrenal function in vivo and in vitro in female rats," Endocrinology, vol. 98, no. 1, pp. 20-24, 1976. 